\title{
The shape of $\beta$-alanine
}

M. Eugenia Sanz, Alberto Lesarri, Isabel Peña, Vanesa Vaquero, Vanessa Cortijo, Juan C. López and José L. Alonso*

\section{Supplementary Information}

Grupo de Espectroscopía Molecular (GEM).

Departamento de Química Física y Química Inorgánica

Facultad de Ciencias, Universidad de Valladolid, 47005 Valladolid (Spain)

E-mail: jlalonso@qf.uva.es; Web: www.uva.es/gem

RECEIVED DATE () 
Complete reference [23]:

Gaussian 03, Revision B.04,

M. J. Frisch, G. W. Trucks, H. B. Schlegel, G. E. Scuseria, M. A. Robb, J. R. Cheeseman, J. A. Montgomery, Jr., T. Vreven, K. N. Kudin, J. C. Burant, J. M. Millam, S. S. Iyengar, J. Tomasi, V. Barone, B. Mennucci, M. Cossi, G. Scalmani, N. Rega, G. A. Petersson, H. Nakatsuji, M. Hada, M. Ehara, K. Toyota, R. Fukuda, J. Hasegawa, M. Ishida, T. Nakajima, Y. Honda, O. Kitao, H. Nakai, M. Klene, X. Li, J. E. Knox, H. P. Hratchian, J. B. Cross, C. Adamo, J. Jaramillo, R. Gomperts, R. E. Stratmann, O. Yazyev, A. J. Austin, R. Cammi, C. Pomelli, J. W. Ochterski, P. Y. Ayala, K. Morokuma, G. A. Voth, P. Salvador, J. J. Dannenberg, V. G. Zakrzewski, S. Dapprich, A. D. Daniels, M. C. Strain, O. Farkas, D. K. Malick, A. D. Rabuck, K. Raghavachari, J. B. Foresman, J. V. Ortiz, Q. Cui, A. G. Baboul, S. Clifford, J. Cioslowski, B. B. Stefanov, G. Liu, A. Liashenko, P. Piskorz, I. Komaromi, R. L. Martin, D. J. Fox, T. Keith, M. A. Al-Laham, C. Y. Peng, A. Nanayakkara, M. Challacombe, P. M. W. Gill, B. Johnson, W. Chen, M. W. Wong, C. Gonzalez, and J. A. Pople, Gaussian, Inc., Pittsburgh PA, 2003. 
Table 1. Predicted spectroscopic parameters and relative energies for the high-energy conformers of $\beta$-alanine from ab-initio MP2/6-311++G(d,p) calculations.

\begin{tabular}{|c|c|c|c|c|c|c|c|c|c|c|}
\hline & VII & VIII & IX & $\mathbf{X}$ & XI & XIII & XIV & XV/ XVIII/XX & XVI/ XIX ${ }^{\mathrm{a}}$ & XVII \\
\hline $\mathrm{A} / \mathrm{MHz}$ & 9182.1 & 7167.5 & 8459.6 & 8209.0 & 6464.4 & 6943.4 & 6965.1 & 9044.7 & 9037.7 & 5582.2 \\
\hline $\mathrm{B} / \mathrm{MHz}$ & 1835.0 & 2404.5 & 1895.0 & 1877.2 & 2448.9 & 2368.0 & 2312.5 & 1859.1 & 1835.3 & 2480.4 \\
\hline $\mathrm{C} / \mathrm{MHz}$ & 1571.6 & 1937.9 & 1668.5 & 1678.1 & 2004.8 & 2047.0 & 1999.0 & 1579.9 & 1567.7 & 2305.2 \\
\hline$\mu_{\mathrm{a}} / \mathrm{D}$ & -0.7 & 1.2 & -1.1 & -0.3 & 0.2 & -1.5 & -0.2 & -2.6 & 1.4 & 1.9 \\
\hline$\mu_{\mathrm{b}} / \mathrm{D}$ & -2.3 & -0.7 & -0.9 & -0.9 & -0.4 & 3.3 & 4.4 & 2.6 & -4.5 & 2.4 \\
\hline$\mu_{\mathrm{c}} / \mathrm{D}$ & 0 & 1.0 & 1.0 & -1.3 & -1.6 & -0.5 & -0.8 & -1.0 & 0 & -2.0 \\
\hline$\mu_{\text {TOTAL }} / \mathrm{D}$ & 2.4 & 1.8 & 1.8 & 1.6 & 1.7 & 3.7 & 4.5 & 3.8 & 4.7 & 3.7 \\
\hline$\chi_{\mathrm{aa}} / \mathrm{MHz}$ & -0.57 & 1.94 & 2.75 & 0.42 & -1.57 & 1.75 & -3.94 & 2.70 & -0.52 & -3.40 \\
\hline$\chi_{\mathrm{bb}} / \mathrm{MHz}$ & -1.49 & 2.41 & 1.69 & 1.66 & 2.32 & 0.49 & 1.69 & -0.76 & -1.54 & 2.18 \\
\hline$\chi_{\mathrm{cc}} / \mathrm{MHz}$ & 2.06 & -4.36 & -4.44 & -2.08 & -0.75 & -2.24 & 2.24 & -1.93 & 2.07 & 1.22 \\
\hline$\Delta \mathrm{E}_{\mathrm{MP} 2} / \mathrm{cm}^{-1}$ & 790 & 676 & 718 & 773 & 755 & 1822 & 2227 & 2535 & 2825 & 2572 \\
\hline$\Delta \mathrm{E}_{\mathrm{MP} 2+\mathrm{ZPC}} / \mathrm{cm}^{-1}$ & 677 & 695 & 706 & 752 & 764 & 1779 & 2133 & 2351 & 2611 & 2507 \\
\hline$\left(\Delta \mathrm{E}_{\mathrm{HF}}\right)^{\mathrm{a}} / \mathrm{cm}^{-1}$ & (677) & (853) & $(869)$ & (953) & (978) & (2274) & (2625) & $(2775) /(3327) /(3603)$ & $(3126) /(3586)$ & $(-)$ \\
\hline
\end{tabular}

${ }^{\mathrm{a}} \mathrm{HF} / 6-31 \mathrm{G}(\mathrm{d}, \mathrm{p})$ relative energies from ref. [4].

${ }^{b}$ The HF/6-31G(d,p) structures of these conformers converged to the same minimum when calculated at MP2 level. 
Table 2. Observed frequencies and residuals (in MHz) for the rotational transitions of conformer I of $\beta$-alanine.

\begin{tabular}{|c|c|c|c|c|c|c|c|c|c|}
\hline$J^{\prime}$ & $K_{-1}^{\prime}$ & $K_{+1}^{\prime}$ & $J^{\prime \prime}$ & $K_{-1}^{\prime \prime}$ & $K_{+1}^{\prime \prime}$ & $F^{\prime}$ & $F^{\prime \prime}$ & $v_{\text {obs }}$ & $v_{\text {obs }}-v_{\text {cal }}$ \\
\hline \multirow[t]{3}{*}{1} & 1 & 1 & 0 & 0 & 0 & 2 & 1 & 9254.564 & 0.000 \\
\hline & & & & & & 1 & 1 & 9254.828 & -0.002 \\
\hline & & & & & & 0 & 1 & 9254.165 & 0.000 \\
\hline \multirow[t]{3}{*}{1} & 1 & 0 & 0 & 0 & 0 & 2 & 1 & 9603.133 & 0.003 \\
\hline & & & & & & 1 & 1 & 9602.343 & -0.002 \\
\hline & & & & & & 0 & 1 & 9604.309 & 0.002 \\
\hline \multirow[t]{5}{*}{2} & 1 & 2 & 1 & 0 & 1 & 3 & 2 & 13228.051 & 0.003 \\
\hline & & & & & & 2 & 1 & 13228.370 & 0.000 \\
\hline & & & & & & 1 & 0 & 13228.351 & -0.008 \\
\hline & & & & & & 2 & 2 & 13228.889 & 0.000 \\
\hline & & & & & & 1 & 1 & 13227.068 & 0.006 \\
\hline \multirow[t]{5}{*}{2} & 1 & 1 & 1 & 0 & 1 & 3 & 2 & 14273.472 & 0.001 \\
\hline & & & & & & 2 & 1 & 14272.667 & -0.001 \\
\hline & & & & & & 1 & 0 & 14274.412 & 0.004 \\
\hline & & & & & & 2 & 2 & 14273.186 & 0.000 \\
\hline & & & & & & 1 & 1 & 14273.106 & -0.005 \\
\hline \multirow[t]{3}{*}{3} & 0 & 3 & 2 & 1 & 2 & 4 & 3 & 8292.746 & -0.002 \\
\hline & & & & & & 3 & 2 & 8292.584 & 0.004 \\
\hline & & & & & & 2 & 1 & 8292.978 & -0.002 \\
\hline \multirow[t]{3}{*}{4} & 0 & 4 & 3 & 1 & 3 & 5 & 4 & 12972.678 & 0.001 \\
\hline & & & & & & 4 & 3 & 12972.416 & 0.002 \\
\hline & & & & & & 3 & 2 & 12972.837 & -0.003 \\
\hline
\end{tabular}


Table 3. Observed frequencies and residuals (in MHz) for the rotational transitions of conformer II of $\beta$-alanine.

\begin{tabular}{|c|c|c|c|c|c|c|c|c|c|}
\hline$J^{\prime}$ & $K_{-1}^{\prime}$ & $K_{+1}^{\prime}$ & $J^{\prime \prime}$ & $K_{-1}^{\prime \prime}$ & $K_{+1}^{\prime \prime}$ & $F^{\prime}$ & $F^{\prime \prime}$ & $v_{\text {obs }}$ & $v_{\text {obs }}-v_{c a l}$ \\
\hline \multirow[t]{3}{*}{1} & 1 & 1 & 0 & 0 & 0 & 2 & 1 & 9134.776 & 0.000 \\
\hline & & & & & & 1 & 1 & 9135.253 & 0.000 \\
\hline & & & & & & 0 & 1 & 9134.058 & 0.000 \\
\hline \multirow[t]{3}{*}{1} & 1 & 0 & 0 & 0 & 0 & 2 & 1 & 9459.136 & 0.000 \\
\hline & & & & & & 1 & 1 & 9459.708 & -0.002 \\
\hline & & & & & & 0 & 1 & 9458.275 & -0.001 \\
\hline \multirow[t]{5}{*}{2} & 0 & 2 & 1 & 0 & 1 & 3 & 2 & 8530.520 & 0.000 \\
\hline & & & & & & 2 & 1 & 8530.443 & 0.000 \\
\hline & & & & & & 1 & 0 & 8529.570 & 0.001 \\
\hline & & & & & & 2 & 2 & 8529.390 & 0.000 \\
\hline & & & & & & 1 & 1 & 8532.198 & -0.001 \\
\hline \multirow[t]{5}{*}{2} & 1 & 2 & 1 & 1 & 1 & 3 & 2 & 8221.988 & 0.001 \\
\hline & & & & & & 2 & 1 & 8220.896 & 0.002 \\
\hline & & & & & & 1 & 0 & 8223.048 & 0.001 \\
\hline & & & & & & 2 & 2 & 8221.372 & -0.001 \\
\hline & & & & & & 1 & 1 & 8221.851 & -0.001 \\
\hline \multirow[t]{5}{*}{2} & 1 & 1 & 1 & 1 & 0 & 3 & 2 & 8870.733 & -0.001 \\
\hline & & & & & & 2 & 1 & 8869.646 & -0.001 \\
\hline & & & & & & 1 & 0 & 8871.879 & -0.001 \\
\hline & & & & & & 2 & 2 & 8870.219 & -0.002 \\
\hline & & & & & & 1 & 1 & 8870.445 & 0.000 \\
\hline \multirow[t]{3}{*}{2} & 1 & 2 & 1 & 0 & 1 & 3 & 2 & 13083.511 & 0.004 \\
\hline & & & & & & 2 & 1 & 13083.943 & -0.002 \\
\hline & & & & & & 1 & 0 & 13082.275 & 0.004 \\
\hline \multirow[t]{3}{*}{2} & 1 & 1 & 1 & 0 & 1 & 3 & 2 & 14056.612 & -0.002 \\
\hline & & & & & & 2 & 1 & 14057.150 & -0.004 \\
\hline & & & & & & 1 & 0 & 14055.318 & -0.002 \\
\hline \multirow[t]{3}{*}{3} & 0 & 3 & 2 & 0 & 2 & 4 & 3 & 12756.660 & 0.000 \\
\hline & & & & & & 3 & 2 & 12756.617 & 0.002 \\
\hline & & & & & & 2 & 1 & 12756.442 & 0.000 \\
\hline \multirow[t]{5}{*}{3} & 1 & 3 & 2 & 1 & 2 & 4 & 3 & 12323.117 & 0.001 \\
\hline & & & & & & 3 & 2 & 12322.800 & -0.001 \\
\hline & & & & & & 2 & 1 & 12323.097 & -0.003 \\
\hline & & & & & & 3 & 3 & 12322.185 & 0.000 \\
\hline & & & & & & 2 & 2 & 12324.058 & 0.001 \\
\hline \multirow[t]{5}{*}{3} & 1 & 2 & 2 & 1 & 1 & 4 & 3 & 13295.769 & 0.002 \\
\hline & & & & & & 3 & 2 & 13295.458 & 0.004 \\
\hline & & & & & & 2 & 1 & 13295.769 & -0.001 \\
\hline & & & & & & 3 & 3 & 13294.945 & 0.003 \\
\hline & & & & & & 2 & 2 & 13296.572 & 0.004 \\
\hline \multirow[t]{5}{*}{3} & 0 & 3 & 2 & 1 & 2 & 4 & 3 & 8203.670 & -0.001 \\
\hline & & & & & & 3 & 2 & 8203.112 & -0.001 \\
\hline & & & & & & 2 & 1 & 8203.742 & 0.001 \\
\hline & & & & & & 3 & 3 & 8202.495 & -0.003 \\
\hline & & & & & & 2 & 2 & 8204.698 & 0.000 \\
\hline \multirow[t]{3}{*}{4} & 0 & 4 & 3 & 0 & 3 & 5 & 4 & 16937.352 & 0.002 \\
\hline & & & & & & 4 & 3 & 16937.321 & 0.002 \\
\hline & & & & & & 3 & 2 & 16937.250 & 0.002 \\
\hline \multirow[t]{3}{*}{4} & 1 & 4 & 3 & 1 & 3 & 5 & 4 & 16413.408 & -0.003 \\
\hline & & & & & & 4 & 3 & 16413.266 & -0.003 \\
\hline & & & & & & 3 & 2 & 16413.359 & -0.001 \\
\hline \multirow[t]{3}{*}{4} & 0 & 4 & 3 & 1 & 3 & 5 & 4 & 12817.907 & 0.002 \\
\hline & & & & & & 4 & 3 & 12817.634 & 0.001 \\
\hline & & & & & & 3 & 2 & 12817.886 & -0.003 \\
\hline
\end{tabular}


Table 4. Observed frequencies and residuals (in MHz) for the rotational transitions of conformer $\mathrm{V}$ of $\beta$-alanine.

\begin{tabular}{|c|c|c|c|c|c|c|c|c|c|}
\hline$J^{\prime}$ & $K_{-1}^{\prime}$ & $K_{+1}^{\prime}$ & $J^{\prime \prime}$ & $K_{-1}^{\prime \prime}$ & $K_{+1}^{\prime \prime}$ & $F^{\prime}$ & $F^{\prime \prime}$ & $v_{\text {obs }}$ & $v_{\text {obs }}-v_{\text {cal }}$ \\
\hline \multirow[t]{3}{*}{1} & 1 & 1 & 0 & 0 & 0 & 2 & 1 & 9159.546 & -0.001 \\
\hline & & & & & & 1 & 1 & 9159.378 & 0.003 \\
\hline & & & & & & 0 & 1 & 9159.810 & 0.004 \\
\hline \multirow[t]{5}{*}{2} & 0 & 2 & 1 & 0 & 1 & 3 & 2 & 8923.805 & 0.000 \\
\hline & & & & & & 2 & 1 & 8923.740 & -0.001 \\
\hline & & & & & & 1 & 0 & 8923.498 & 0.002 \\
\hline & & & & & & 2 & 2 & 8923.377 & 0.001 \\
\hline & & & & & & 1 & 1 & 8924.409 & 0.001 \\
\hline \multirow[t]{5}{*}{2} & 1 & 2 & 1 & 1 & 1 & 3 & 2 & 8447.178 & -0.001 \\
\hline & & & & & & 2 & 1 & 8446.772 & -0.003 \\
\hline & & & & & & 1 & 0 & 8447.241 & 0.002 \\
\hline & & & & & & 2 & 2 & 8446.603 & 0.000 \\
\hline & & & & & & 1 & 1 & 8446.670 & 0.000 \\
\hline \multirow[t]{5}{*}{2} & 1 & 1 & 1 & 1 & 0 & 3 & 2 & 9481.654 & 0.002 \\
\hline & & & & & & 2 & 1 & 9481.305 & 0.005 \\
\hline & & & & & & 1 & 0 & 9482.354 & -0.001 \\
\hline & & & & & & 2 & 2 & 9481.834 & -0.003 \\
\hline & & & & & & 1 & 1 & 9481.014 & 0.001 \\
\hline \multirow[t]{3}{*}{2} & 1 & 2 & 1 & 0 & 1 & 3 & 2 & 13124.485 & -0.005 \\
\hline & & & & & & 2 & 1 & 13124.281 & 0.002 \\
\hline & & & & & & 1 & 0 & 13124.259 & -0.003 \\
\hline \multirow[t]{5}{*}{3} & 0 & 3 & 2 & 0 & 2 & 4 & 3 & 13285.871 & 0.001 \\
\hline & & & & & & 3 & 2 & 13285.796 & -0.001 \\
\hline & & & & & & 2 & 1 & 13285.805 & -0.003 \\
\hline & & & & & & 3 & 3 & 13285.366 & -0.003 \\
\hline & & & & & & 2 & 2 & 13286.473 & -0.002 \\
\hline \multirow[t]{4}{*}{3} & 1 & 3 & 2 & 1 & 2 & 4 & 3 & 12646.215 & 0.002 \\
\hline & & & & & & 3 & 2 & 12646.079 & 0.000 \\
\hline & & & & & & 3 & 3 & 12645.508 & 0.004 \\
\hline & & & & & & 2 & 2 & 12647.040 & 0.004 \\
\hline \multirow[t]{3}{*}{3} & 1 & 2 & 2 & 1 & 1 & 4 & 3 & 14195.974 & -0.003 \\
\hline & & & & & & 3 & 2 & 14195.868 & -0.001 \\
\hline & & & & & & 2 & 1 & 14196.054 & 0.002 \\
\hline \multirow[t]{3}{*}{3} & 0 & 3 & 2 & 1 & 2 & 4 & 3 & 9085.186 & 0.000 \\
\hline & & & & & & 3 & 2 & 9085.261 & 0.003 \\
\hline & & & & & & 2 & 1 & 9085.036 & -0.006 \\
\hline \multirow[t]{3}{*}{4} & 0 & 4 & 3 & 1 & 3 & 5 & 4 & 13976.108 & -0.001 \\
\hline & & & & & & 4 & 3 & 13976.236 & 0.004 \\
\hline & & & & & & 3 & 2 & 13976.011 & -0.001 \\
\hline
\end{tabular}


Table 5. Observed frequencies and residuals (in MHz) for the rotational transitions of conformer III of $\beta$-alanine.

\begin{tabular}{|c|c|c|c|c|c|c|c|c|c|}
\hline$J^{\prime}$ & $K_{-1}^{\prime}$ & $K_{+1}^{\prime}$ & $J^{\prime \prime}$ & $K_{-1}^{\prime \prime}$ & $K_{+1}^{\prime \prime}$ & $F^{\prime}$ & $F^{\prime \prime}$ & $v_{\text {obs }}$ & $v_{\text {obs }}-v_{\text {cal }}$ \\
\hline \multirow[t]{3}{*}{1} & 0 & 1 & 0 & 0 & 0 & 2 & 1 & 4738.2636 & -0.002 \\
\hline & & & & & & 1 & 1 & 4737.5447 & 0.001 \\
\hline & & & & & & 0 & 1 & 4739.3482 & 0.000 \\
\hline \multirow[t]{5}{*}{2} & 0 & 2 & 1 & 0 & 1 & 3 & 2 & 9473.0535 & 0.001 \\
\hline & & & & & & 2 & 1 & 9473.0089 & -0.001 \\
\hline & & & & & & 1 & 0 & 9472.3982 & 0.003 \\
\hline & & & & & & 2 & 2 & 9472.2890 & 0.000 \\
\hline & & & & & & 1 & 1 & 9474.2001 & 0.002 \\
\hline \multirow[t]{3}{*}{2} & 1 & 2 & 1 & 1 & 1 & 3 & 2 & 9353.9316 & 0.002 \\
\hline & & & & & & 2 & 1 & 9353.2010 & 0.002 \\
\hline & & & & & & 1 & 0 & 9354.8980 & 0.000 \\
\hline \multirow[t]{3}{*}{2} & 1 & 1 & 1 & 1 & 0 & 3 & 2 & 9598.8421 & -0.002 \\
\hline & & & & & & 2 & 1 & 9598.0754 & -0.005 \\
\hline & & & & & & 1 & 0 & 9599.3848 & -0.002 \\
\hline \multirow[t]{3}{*}{3} & 0 & 3 & 2 & 0 & 2 & 4 & 3 & 14201.3273 & 0.002 \\
\hline & & & & & & 3 & 2 & 14201.3093 & -0.002 \\
\hline & & & & & & 2 & 1 & 14201.1725 & -0.001 \\
\hline \multirow[t]{3}{*}{3} & 1 & 3 & 2 & 1 & 2 & 4 & 3 & 14028.6310 & -0.001 \\
\hline & & & & & & 3 & 2 & 14028.4283 & -0.001 \\
\hline & & & & & & 2 & 1 & 14028.6729 & -0.002 \\
\hline \multirow[t]{3}{*}{3} & 1 & 2 & 2 & 1 & 1 & 4 & 3 & 14395.9188 & 0.000 \\
\hline & & & & & & 3 & 2 & 14395.6980 & 0.004 \\
\hline & & & & & & 2 & 1 & 14395.8692 & 0.005 \\
\hline
\end{tabular}


Table 6. Ab initio structure at the MP2/6-311++G(d,p) level of theory for conformer I of $\beta$-alanine.

\begin{tabular}{rrrrrrrr}
\hline Cent & Atom & N1 & Length $/ \AA$ & N2 & $\alpha /$ degrees & N3 & $\beta /$ degrees \\
\hline 1 & $\mathrm{C}$ & & & & & & \\
2 & $\mathrm{C}$ & 1 & 1.5232 & & & & \\
3 & $\mathrm{~N}$ & 2 & 1.4649 & 1 & 109.92 & & \\
4 & $\mathrm{C}$ & 1 & 1.5077 & 2 & 111.99 & 3 & -64.31 \\
5 & $\mathrm{O}$ & 4 & 1.3557 & 1 & 111.55 & 2 & 174.23 \\
6 & $\mathrm{O}$ & 4 & 1.2130 & 1 & 125.79 & 2 & -6.44 \\
7 & $\mathrm{H}$ & 3 & 1.0146 & 2 & 110.24 & 1 & -166.01 \\
8 & $\mathrm{H}$ & 3 & 1.0154 & 2 & 109.24 & 1 & 76.48 \\
9 & $\mathrm{H}$ & 5 & 0.9685 & 4 & 105.95 & 1 & -180.66 \\
10 & $\mathrm{H}$ & 2 & 1.0992 & 1 & 109.09 & 4 & 60.77 \\
11 & $\mathrm{H}$ & 2 & 1.0939 & 1 & 108.19 & 4 & 177.71 \\
12 & $\mathrm{H}$ & 1 & 1.0943 & 4 & 108.24 & 6 & -128.26 \\
13 & $\mathrm{H}$ & 1 & 1.0965 & 4 & 107.41 & 6 & 115.96 \\
& & & & & & & \\
\hline
\end{tabular}

Table 7. Ab initio structure at the MP2/6-311++G(d,p) level of theory for conformer II of $\beta$-alanine.

\begin{tabular}{rrrrrrrr}
\hline Cent & Atom & N1 & Length/ $\AA$ & N2 & $\alpha /$ degrees & N3 & $\beta /$ degrees \\
\hline 1 & $\mathrm{C}$ & & & & & & \\
2 & $\mathrm{C}$ & 1 & 1.5313 & & & & \\
3 & $\mathrm{~N}$ & 2 & 1.4590 & 1 & 115.75 & & \\
4 & $\mathrm{C}$ & 1 & 1.5084 & 2 & 112.25 & 3 & -63.23 \\
5 & $\mathrm{O}$ & 4 & 1.3568 & 1 & 111.60 & 2 & 168.37 \\
6 & $\mathrm{O}$ & 4 & 1.2123 & 1 & 125.64 & 2 & -12.33 \\
7 & $\mathrm{H}$ & 3 & 1.0152 & 2 & 110.86 & 1 & -56.41 \\
8 & $\mathrm{H}$ & 3 & 1.0146 & 2 & 110.08 & 1 & 62.93 \\
9 & $\mathrm{H}$ & 5 & 0.9687 & 4 & 105.96 & 1 & 178.13 \\
10 & $\mathrm{H}$ & 2 & 1.0932 & 1 & 109.08 & 4 & 58.45 \\
11 & $\mathrm{H}$ & 2 & 1.0945 & 1 & 108.36 & 4 & 174.92 \\
12 & $\mathrm{H}$ & 1 & 1.0955 & 4 & 108.40 & 6 & -135.65 \\
13 & $\mathrm{H}$ & 1 & 1.0978 & 4 & 107.15 & 6 & 109.66 \\
& & & & & & & \\
\hline
\end{tabular}

Table 8. Ab initio structure at the MP2/6-311++G(d,p) level of theory for conformer III of $\beta$-alanine.

\begin{tabular}{rrrrrrrr}
\hline Cent & Atom & N1 & Length $/ \AA$ & N2 & $\alpha /$ degrees & N3 & $\beta /$ degrees \\
\hline 1 & $\mathrm{C}$ & & & & & & \\
2 & $\mathrm{C}$ & 1 & 1.5286 & & & & \\
3 & $\mathrm{~N}$ & 2 & 1.4639 & 1 & 108.90 & & \\
4 & $\mathrm{C}$ & 1 & 1.5087 & 2 & 111.25 & 3 & -53.90 \\
5 & $\mathrm{O}$ & 4 & 1.3588 & 1 & 111.00 & 2 & -57.84 \\
6 & $\mathrm{O}$ & 4 & 1.2098 & 1 & 126.03 & 2 & 123.10 \\
7 & $\mathrm{H}$ & 3 & 1.0161 & 2 & 110.46 & 1 & -66.53 \\
8 & $\mathrm{H}$ & 3 & 1.0148 & 2 & 110.80 & 1 & 174.88 \\
9 & $\mathrm{H}$ & 5 & 0.9681 & 4 & 105.70 & 1 & 178.34 \\
10 & $\mathrm{H}$ & 2 & 1.1001 & 1 & 108.59 & 4 & -178.82 \\
11 & $\mathrm{H}$ & 2 & 1.0926 & 1 & 109.24 & 4 & 63.72 \\
12 & $\mathrm{H}$ & 1 & 1.0954 & 4 & 108.82 & 6 & -115.71 \\
13 & $\mathrm{H}$ & 1 & 1.0929 & 4 & 107.41 & 6 & 1.54 \\
& & & & & & & \\
\hline
\end{tabular}


Table 9. Ab initio structure at the MP2/6-311++G(d,p) level of theory for conformer IV/XII of $\beta$-alanine.

\begin{tabular}{rrrrrrrr}
\hline Cent & Atom & N1 & Length/ $\AA$ & N2 & $\alpha /$ degrees & N3 & $\beta /$ degrees \\
\hline 1 & $\mathrm{C}$ & & & & & & \\
2 & $\mathrm{C}$ & 1 & 1.5225 & & & & \\
3 & $\mathrm{~N}$ & 2 & 1.4660 & 1 & 109.08 & & \\
4 & $\mathrm{C}$ & 1 & 1.5064 & 2 & 112.39 & 3 & -175.49 \\
5 & $\mathrm{O}$ & 4 & 1.3584 & 1 & 111.38 & 2 & -168.70 \\
6 & $\mathrm{O}$ & 4 & 1.2113 & 1 & 125.79 & 2 & 12.18 \\
7 & $\mathrm{H}$ & 3 & 1.0152 & 2 & 110.18 & 1 & 175.32 \\
8 & $\mathrm{H}$ & 3 & 1.0159 & 2 & 110.11 & 1 & -67.23 \\
9 & $\mathrm{H}$ & 5 & 0.9685 & 4 & 105.89 & 1 & -177.97 \\
10 & $\mathrm{H}$ & 2 & 1.0992 & 1 & 109.12 & 4 & 59.32 \\
11 & $\mathrm{H}$ & 2 & 1.0925 & 1 & 109.04 & 4 & -57.30 \\
12 & $\mathrm{H}$ & 1 & 1.0955 & 4 & 108.48 & 6 & 135.74 \\
13 & $\mathrm{H}$ & 1 & 1.0958 & 4 & 108.13 & 6 & -109.09 \\
& & & & & & & \\
\hline
\end{tabular}

Table 10. Ab initio structure at the MP2/6-311++G(d,p) level of theory for conformer $V$ of $\beta$-alanine.

\begin{tabular}{rrrrrrrr}
\hline Cent & Atom & N1 & Length/ $\AA$ & N2 & $\alpha /$ degrees & N3 & $\beta /$ degrees \\
\hline 1 & $\mathrm{C}$ & & & & & & \\
2 & $\mathrm{C}$ & 1 & 1.5301 & & & & \\
3 & $\mathrm{~N}$ & 2 & 1.4757 & 1 & 109.56 & & \\
4 & $\mathrm{C}$ & 1 & 1.5280 & 2 & 114.32 & 3 & -64.65 \\
5 & $\mathrm{O}$ & 4 & 1.3457 & 1 & 115.43 & 2 & 39.89 \\
6 & $\mathrm{O}$ & 4 & 1.2095 & 1 & 122.61 & 2 & -141.28 \\
7 & $\mathrm{H}$ & 3 & 1.0169 & 2 & 110.27 & 1 & -74.83 \\
8 & $\mathrm{H}$ & 3 & 1.0157 & 2 & 111.01 & 1 & 166.81 \\
9 & $\mathrm{H}$ & 5 & 0.9878 & 4 & 107.66 & 1 & -3.72 \\
10 & $\mathrm{H}$ & 2 & 1.0942 & 1 & 109.53 & 4 & 53.12 \\
11 & $\mathrm{H}$ & 2 & 1.0967 & 1 & 109.52 & 4 & 171.05 \\
12 & $\mathrm{H}$ & 1 & 1.0981 & 4 & 107.73 & 6 & 97.01 \\
13 & $\mathrm{H}$ & 1 & 1.0918 & 4 & 107.19 & 6 & -18.30 \\
& & & & & & & \\
\hline
\end{tabular}

Table 11. Ab initio structure at the MP2/6-311++G(d,p) level of theory for conformer VI of $\beta$-alanine.

\begin{tabular}{rrrrrrrr}
\hline Cent & Atom & $\mathrm{N} 1$ & Length $/ \AA$ & $\mathrm{N} 2$ & $\alpha /$ degrees & $\mathrm{N} 3$ & $\beta /$ degrees \\
\hline & & & & & & & \\
1 & $\mathrm{C}$ & & & & & & \\
3 & $\mathrm{C}$ & 1 & 1.5400 & & & & \\
4 & $\mathrm{~N}$ & 2 & 1.4589 & 1 & 115.50 & & \\
5 & $\mathrm{C}$ & 1 & 1.5056 & 2 & 111.83 & 3 & -55.69 \\
6 & $\mathrm{O}$ & 4 & 1.3616 & 1 & 111.06 & 2 & -56.29 \\
7 & $\mathrm{O}$ & 4 & 1.2108 & 1 & 126.38 & 2 & 122.62 \\
8 & $\mathrm{H}$ & 3 & 1.0151 & 2 & 111.03 & 1 & 66.39 \\
9 & $\mathrm{H}$ & 3 & 1.0155 & 2 & 111.01 & 1 & -52.95 \\
10 & $\mathrm{H}$ & 5 & 0.9686 & 4 & 105.87 & 1 & -179.56 \\
11 & $\mathrm{H}$ & 2 & 1.0945 & 1 & 108.17 & 4 & -177.13 \\
12 & $\mathrm{H}$ & 2 & 1.0926 & 1 & 109.37 & 4 & 66.27 \\
13 & $\mathrm{H}$ & 1 & 1.0966 & 4 & 108.42 & 6 & -116.39 \\
& $\mathrm{H}$ & 1 & 1.0927 & 4 & 107.36 & 6 & 0.90 \\
& & & & & & & \\
\hline
\end{tabular}


Table 12. Ab initio structure at the MP2/6-311++G(d,p) level of theory for conformer VII of $\beta$-alanine.

\begin{tabular}{rrrrrrrr}
\hline Cent & Atom & N1 & Length/ $\AA$ & N2 & $\alpha /$ degrees & N3 & $\beta /$ degrees \\
\hline 1 & $\mathrm{C}$ & & & & & & \\
2 & $\mathrm{C}$ & 1 & 1.5311 & & & & \\
3 & $\mathrm{~N}$ & 2 & 1.4617 & 1 & 114.81 & & \\
4 & $\mathrm{C}$ & 1 & 1.5081 & 2 & 112.75 & 3 & 180.00 \\
5 & $\mathrm{O}$ & 4 & 1.3590 & 1 & 111.34 & 2 & 180.00 \\
6 & $\mathrm{O}$ & 4 & 1.2109 & 1 & 125.7 & 2 & 0.00 \\
7 & $\mathrm{H}$ & 3 & 1.0154 & 2 & 110.63 & 1 & -59.22 \\
8 & $\mathrm{H}$ & 3 & 1.0154 & 2 & 110.63 & 1 & 59.22 \\
9 & $\mathrm{H}$ & 5 & 0.9685 & 4 & 105.92 & 1 & 180.00 \\
10 & $\mathrm{H}$ & 2 & 1.0931 & 1 & 109.29 & 4 & -58.05 \\
11 & $\mathrm{H}$ & 2 & 1.0931 & 1 & 109.29 & 4 & 58.05 \\
12 & $\mathrm{H}$ & 1 & 1.0965 & 4 & 107.93 & 6 & -122.74 \\
13 & $\mathrm{H}$ & 1 & 1.0965 & 4 & 107.93 & 6 & 122.74 \\
& & & & & & & \\
\hline
\end{tabular}

Table 13. Ab initio structure at the MP2/6-311++G(d,p) level of theory for conformer VIII of $\beta$-alanine.

\begin{tabular}{rrrrrrrr}
\hline Cent & Atom & N1 & Length/ $\AA$ & N2 & $\alpha /$ degrees & N3 & $\beta /$ degrees \\
\hline & & & & & & & \\
2 & $\mathrm{C}$ & & & & & & \\
3 & $\mathrm{C}$ & 1 & 1.5342 & & & & \\
4 & $\mathrm{~N}$ & 2 & 1.4636 & 1 & 111.30 & & \\
5 & $\mathrm{C}$ & 1 & 1.5069 & 2 & 113.74 & 3 & -80.20 \\
6 & $\mathrm{O}$ & 4 & 1.3646 & 1 & 111.82 & 2 & 52.54 \\
7 & $\mathrm{O}$ & 4 & 1.2103 & 1 & 126.07 & 2 & -127.23 \\
8 & $\mathrm{H}$ & 3 & 1.0149 & 2 & 109.92 & 1 & 66.42 \\
9 & $\mathrm{H}$ & 3 & 1.0152 & 2 & 109.88 & 1 & -175.68 \\
10 & $\mathrm{H}$ & 5 & 0.9688 & 4 & 105.79 & 1 & 180.21 \\
11 & $\mathrm{H}$ & 2 & 1.0940 & 1 & 107.59 & 4 & 162.11 \\
12 & $\mathrm{H}$ & 2 & 1.1016 & 1 & 108.86 & 4 & 46.30 \\
13 & $\mathrm{H}$ & 1 & 1.0917 & 4 & 106.49 & 6 & -5.07 \\
& & & 1.0957 & 4 & 108.62 & 6 & 112.13 \\
\hline
\end{tabular}

Table 14. Ab initio structure at the MP2/6-311++G(d,p) level of theory for conformer IX of $\beta$-alanine.

\begin{tabular}{rrrrrrrr}
\hline Cent & Atom & N1 & Length/ $\AA$ & N2 & $\alpha /$ degrees & N3 & $\beta /$ degrees \\
\hline & & & & & & & \\
2 & $\mathrm{C}$ & & & & & & \\
3 & $\mathrm{C}$ & 1 & 1.5313 & & & & \\
4 & $\mathrm{~N}$ & 2 & 1.4644 & 1 & 108.98 & & \\
5 & $\mathrm{C}$ & 1 & 1.5054 & 2 & 111.82 & 3 & -177.83 \\
6 & $\mathrm{O}$ & 4 & 1.3604 & 1 & 111.11 & 2 & -60.95 \\
7 & $\mathrm{O}$ & 4 & 1.2111 & 1 & 126.25 & 2 & 118.18 \\
8 & $\mathrm{H}$ & 3 & 1.0152 & 2 & 110.33 & 1 & -173.97 \\
9 & $\mathrm{H}$ & 3 & 1.0159 & 2 & 110.05 & 1 & 68.35 \\
10 & $\mathrm{H}$ & 5 & 0.9685 & 4 & 105.87 & 1 & -180.54 \\
11 & $\mathrm{H}$ & 2 & 1.1011 & 1 & 108.92 & 4 & -53.17 \\
12 & $\mathrm{H}$ & 2 & 1.0925 & 1 & 109.14 & 4 & 64.05 \\
13 & $\mathrm{H}$ & 1 & 1.0926 & 4 & 107.43 & 6 & -3.19 \\
& $\mathrm{H}$ & 1 & 1.0942 & 4 & 109.45 & 6 & -121.26 \\
\end{tabular}


Table 15. Ab initio structure at the MP2/6-311++G(d,p) level of theory for conformer $X$ of $\beta$-alanine.

\begin{tabular}{rrrrrrrr}
\hline Cent & Atom & N1 & Length/ $\AA$ & N2 & $\alpha /$ degrees & N3 & $\beta /$ degrees \\
\hline & & & & & & & \\
1 & $\mathrm{C}$ & & & & & & \\
3 & $\mathrm{C}$ & 1 & 1.5445 & & & & \\
4 & $\mathrm{~N}$ & 2 & 1.4581 & 1 & 115.06 & & \\
5 & $\mathrm{C}$ & 1 & 1.5047 & 2 & 110.62 & 3 & -177.80 \\
6 & $\mathrm{O}$ & 4 & 1.3603 & 1 & 111.14 & 2 & -75.53 \\
7 & $\mathrm{O}$ & 4 & 1.2113 & 1 & 126.01 & 2 & 101.78 \\
8 & $\mathrm{H}$ & 3 & 1.01523 & 2 & 110.68 & 1 & -59.90 \\
9 & $\mathrm{H}$ & 3 & 1.0152 & 2 & 110.72 & 1 & 58.93 \\
10 & $\mathrm{H}$ & 5 & 0.9686 & 4 & 105.95 & 1 & 176.73 \\
11 & $\mathrm{H}$ & 2 & 1.0949 & 1 & 108.85 & 4 & -56.42 \\
12 & $\mathrm{H}$ & 2 & 1.0935 & 1 & 109.03 & 4 & 60.37 \\
13 & $\mathrm{H}$ & 1 & 1.0924 & 4 & 107.91 & 6 & -18.53 \\
& $\mathrm{H}$ & 1 & 1.0943 & 4 & 109.55 & 6 & -137.65 \\
\hline
\end{tabular}

Table 16. Ab initio structure at the MP2/6-311++G(d,p) level of theory for conformer XI of $\beta$-alanine.

\begin{tabular}{rrrrrrrr}
\hline Cent & Atom & N1 & Length/ $\AA$ & N2 & $\alpha /$ degrees & N3 & $\beta /$ degrees \\
\hline 1 & $\mathrm{C}$ & & & & & & \\
2 & $\mathrm{C}$ & 1 & 1.5488 & & & & \\
3 & $\mathrm{~N}$ & 2 & 1.4574 & 1 & 116.84 & & \\
4 & $\mathrm{C}$ & 1 & 1.5043 & 2 & 111.66 & 3 & -74.08 \\
5 & $\mathrm{O}$ & 4 & 1.3648 & 1 & 111.76 & 2 & 71.98 \\
6 & $\mathrm{O}$ & 4 & 1.2108 & 1 & 125.97 & 2 & -105.71 \\
7 & $\mathrm{H}$ & 3 & 1.0149 & 2 & 110.37 & 1 & 53.43 \\
8 & $\mathrm{H}$ & 3 & 1.0154 & 2 & 110.12 & 1 & -64.02 \\
9 & $\mathrm{H}$ & 5 & 0.9692 & 4 & 105.66 & 1 & 182.00 \\
10 & $\mathrm{H}$ & 2 & 1.0941 & 1 & 107.74 & 4 & 164.29 \\
11 & $\mathrm{H}$ & 2 & 1.0954 & 1 & 108.71 & 4 & 48.99 \\
12 & $\mathrm{H}$ & 1 & 1.0926 & 4 & 106.98 & 6 & 14.54 \\
13 & $\mathrm{H}$ & 1 & 1.0946 & 4 & 109.66 & 6 & 133.02 \\
& & & & & & & \\
\hline
\end{tabular}

Table 17. Ab initio structure at the MP2/6-311++G(d,p) level of theory for conformer XIII of $\beta$-alanine.

\begin{tabular}{rrrrrrrr}
\hline Cent & Atom & N1 & Length/ $\AA$ & N2 & $\alpha /$ degrees & N3 & $\beta /$ degrees \\
\hline & & & & & & & \\
2 & $\mathrm{C}$ & & & & & & \\
3 & $\mathrm{C}$ & 1 & 1.5237 & & & & \\
4 & $\mathrm{~N}$ & 2 & 1.4640 & 1 & 109.44 & & \\
5 & $\mathrm{C}$ & 1 & 1.5190 & 2 & 111.56 & 3 & -63.86 \\
6 & $\mathrm{O}$ & 4 & 1.3614 & 1 & 115.48 & 2 & 168.87 \\
7 & $\mathrm{O}$ & 4 & 1.2063 & 1 & 124.45 & 2 & -12.68 \\
8 & $\mathrm{H}$ & 3 & 1.0145 & 2 & 110.29 & 1 & -165.22 \\
9 & $\mathrm{H}$ & 3 & 1.0157 & 2 & 109.29 & 1 & 77.26 \\
10 & $\mathrm{H}$ & 5 & 0.9638 & 4 & 108.76 & 1 & -4.88 \\
11 & $\mathrm{H}$ & 2 & 1.0983 & 1 & 108.87 & 4 & 60.93 \\
12 & $\mathrm{H}$ & 2 & 1.0940 & 1 & 108.38 & 4 & 178.13 \\
13 & $\mathrm{H}$ & 1 & 1.0963 & 4 & 109.46 & 6 & -133.76 \\
& $\mathrm{H}$ & 1 & 1.0979 & 4 & 107.55 & 6 & 108.99 \\
& & & & & & & \\
\hline
\end{tabular}


Table 18. Ab initio structure at the MP2/6-311++G(d,p) level of theory for conformer XIV of $\beta$-alanine.

\begin{tabular}{rrrrrrrr}
\hline Cent & Atom & N1 & Length $/ \AA$ & N2 & $\alpha /$ degrees & N3 & $\beta /$ degrees \\
\hline & & & & & & & \\
2 & $\mathrm{C}$ & & & & & & \\
3 & $\mathrm{C}$ & 1 & 1.5334 & & & & \\
4 & $\mathrm{~N}$ & 2 & 1.4564 & 1 & 115.76 & & \\
5 & $\mathrm{C}$ & 1 & 1.5196 & 2 & 112.12 & 3 & -63.23 \\
6 & $\mathrm{O}$ & 4 & 1.3628 & 1 & 115.52 & 2 & 170.00 \\
7 & $\mathrm{O}$ & 4 & 1.2056 & 1 & 124.46 & 2 & -11.03 \\
8 & $\mathrm{H}$ & 3 & 1.0150 & 2 & 111.21 & 1 & -57.40 \\
9 & $\mathrm{H}$ & 3 & 1.0150 & 2 & 110.19 & 1 & 62.57 \\
10 & $\mathrm{H}$ & 5 & 0.9638 & 4 & 108.86 & 1 & -2.28 \\
11 & $\mathrm{H}$ & 2 & 1.0929 & 1 & 108.68 & 4 & 58.32 \\
12 & $\mathrm{H}$ & 2 & 1.0946 & 1 & 108.36 & 4 & 174.74 \\
13 & $\mathrm{H}$ & 1 & 1.0982 & 4 & 109.22 & 6 & -133.39 \\
& $\mathrm{H}$ & 1 & 1.0990 & 4 & 107.65 & 6 & 110.65 \\
& & & & & & & \\
\hline
\end{tabular}

Table 19. Ab initio structure at the MP2/6-311++G(d,p) level of theory for conformer XV/XVIII/XX of $\beta$-alanine.

\begin{tabular}{rrrrrrrr}
\hline Cent & Atom & N1 & Length $/ \AA$ & N2 & $\alpha /$ degrees & N3 & $\beta /$ degrees \\
\hline & & & & & & & \\
1 & $\mathrm{C}$ & & & & & & \\
3 & $\mathrm{C}$ & 1 & 1.5233 & & & & \\
4 & $\mathrm{~N}$ & 2 & 1.4662 & 1 & 109.12 & & \\
5 & $\mathrm{C}$ & 1 & 1.5173 & 2 & 112.46 & 3 & -176.21 \\
6 & $\mathrm{O}$ & 4 & 1.3647 & 1 & 115.13 & 2 & -181.91 \\
7 & $\mathrm{O}$ & 4 & 1.2047 & 1 & 124.75 & 2 & -2.13 \\
8 & $\mathrm{H}$ & 3 & 1.0160 & 2 & 110.19 & 1 & -69.99 \\
9 & $\mathrm{H}$ & 3 & 1.0152 & 2 & 110.13 & 1 & 172.65 \\
10 & $\mathrm{H}$ & 5 & 0.9637 & 4 & 108.93 & 1 & -1.94 \\
11 & $\mathrm{H}$ & 2 & 1.0977 & 1 & 109.04 & 4 & 58.29 \\
12 & $\mathrm{H}$ & 2 & 1.0927 & 1 & 108.85 & 4 & -58.19 \\
13 & $\mathrm{H}$ & 1 & 1.0967 & 4 & 109.49 & 6 & -123.75 \\
& $\mathrm{H}$ & 1 & 1.0984 & 4 & 108.31 & 6 & 120.01 \\
\hline
\end{tabular}

Table 20. Ab initio structure at the MP2/6-311++G(d,p) level of theory for conformer XVI/XIX of $\beta$-alanine.

\begin{tabular}{rrrrrrrr}
\hline Cent & Atom & N1 & Length $/ \AA$ & N2 & $\alpha /$ degrees & N3 & $\beta /$ degrees \\
\hline & & & & & & & \\
1 & $\mathrm{C}$ & & & & & & \\
3 & $\mathrm{C}$ & 1 & 1.5329 & & & & \\
4 & $\mathrm{~N}$ & 2 & 1.4603 & 1 & 115.06 & & \\
5 & $\mathrm{C}$ & 1 & 1.5196 & 2 & 112.60 & 3 & 180.00 \\
6 & $\mathrm{O}$ & 4 & 1.3654 & 1 & 115.34 & 2 & 180.00 \\
7 & $\mathrm{O}$ & 4 & 1.2042 & 1 & 124.57 & 2 & 0.00 \\
8 & $\mathrm{H}$ & 3 & 1.0152 & 2 & 110.95 & 1 & -59.59 \\
9 & $\mathrm{H}$ & 3 & 1.0152 & 2 & 110.95 & 1 & 59.59 \\
10 & $\mathrm{H}$ & 5 & 0.9636 & 4 & 108.98 & 1 & 0.00 \\
11 & $\mathrm{H}$ & 2 & 1.0927 & 1 & 108.99 & 4 & -57.90 \\
12 & $\mathrm{H}$ & 2 & 1.0927 & 1 & 108.99 & 4 & 57.90 \\
13 & $\mathrm{H}$ & 1 & 1.0983 & 4 & 108.58 & 6 & -122.09 \\
& $\mathrm{H}$ & 1 & 1.0983 & 4 & 108.58 & 6 & 122.09 \\
& & & & & & &
\end{tabular}


Table 21. Ab initio structure at the MP2/6-311++G(d,p) level of theory for conformer XVII of $\beta$-alanine.

\begin{tabular}{rrrrrrrr}
\hline Cent & Atom & N1 & Length/ $\AA$ & N2 & $\alpha /$ degrees & N3 & $\beta /$ degrees \\
\hline & & & & & & & \\
1 & $\mathrm{C}$ & & & & & & \\
3 & $\mathrm{C}$ & 1 & 1.5440 & & & & \\
4 & $\mathrm{~N}$ & 2 & 1.4561 & 1 & 115.63 & & \\
5 & $\mathrm{C}$ & 1 & 1.5145 & 2 & 111.17 & 3 & -54.09 \\
6 & $\mathrm{O}$ & 4 & 1.3668 & 1 & 115.18 & 2 & -64.92 \\
7 & $\mathrm{O}$ & 4 & 1.2051 & 1 & 124.93 & 2 & 112.23 \\
8 & $\mathrm{H}$ & 3 & 1.0156 & 2 & 111.30 & 1 & 63.80 \\
9 & $\mathrm{H}$ & 3 & 1.0155 & 2 & 110.75 & 1 & -55.04 \\
10 & $\mathrm{H}$ & 5 & 0.9639 & 4 & 109.16 & 1 & -3.81 \\
11 & $\mathrm{H}$ & 2 & 1.0942 & 1 & 108.20 & 4 & -175.85 \\
12 & $\mathrm{H}$ & 2 & 1.0961 & 1 & 110.32 & 4 & 68.28 \\
13 & $\mathrm{H}$ & 1 & 1.0979 & 4 & 109.99 & 6 & -125.69 \\
& $\mathrm{H}$ & 1 & 1.0921 & 4 & 107.16 & 6 & -8.06 \\
\hline
\end{tabular}

